\title{
False Positive Diagnosis of Intracranial Aneurysm in the Context of Abusive Head Trauma
}

Yael Braunschweig, MD, PhD, MPH; Bethany A. Mohr, MD;

Hemant A. Parmar, MD; Kimberly K. Monroe, MD, MS

\section{ABSTRACT}

\section{Introduction}

The diagnosis of abusive head trauma (AHT) involves an extensive workup that commonly includes brain computed tomography angiography (CTA) or magnetic resonance angiography (MRA). Digital subtraction angiography (DSA) provides high-resolution imaging of cerebral vasculature and is the gold standard for the diagnosis of cerebral aneurysm, but its invasiveness typically limits its use in the diagnosis of AHT. This report describes a case in which both CTA and MRA falsely indicated the presence of a cerebral aneurysm and how DSA ruled out vascular abnormality, facilitating a diagnosis of AHT.

\section{Case Presentation}

A previously healthy infant with an unremarkable birth and health history presented with vomiting and irritability and soon developed a tense, bulging anterior fontanelle. A cerebral aneurysm observed on CTA and MRA in the context of bilateral mixed attenuation/ intensity subdural fluid collections, cerebral diffusion abnormality, and retinal hemorrhages $(\mathrm{RH})$ prompted a diagnostic workup that encompassed vascular, metabolic, hematologic and traumatic causes. Following an extensive workup, DSA was performed and revealed no evidence of intracranial aneurysm or other vascular abnormality. The constellation of imaging findings, clinical presentation, and the results of the workup led to the diagnosis of AHT.

\section{Discussion}

This case highlights how a tortuous vessel may mimic an intracranial aneurysm on CTA/MRA and how DSA may play a role in the evaluation of suspected AHT by enabling vascular abnormality to be ruled out.

\section{Conclusion}

The false positive rates for diagnosis of cerebral aneurysm using CTA and MRA should be considered when interpreting imaging results positive for cerebral aneurysm in the context of a differential diagnosis that encompasses AHT. 


\section{Introduction}

dentifying victims of abusive head trauma (AHT) is crucial and often involves an extensive workup in order to evaluate for non-abusive etiologies. Missed cases of AHT place victims at significant risk for further injury and possible death within the environment in which the AHT occurred. ${ }^{1}$ In the United States, the incidence of AHT is estimated to be 39.8 per 100,000 among children less than 1 year of age, with a mortality rate between $13-35 \% .^{1-3}$ Among survivors of pediatric AHT, neurologic, cognitive, and physical impairments are common. ${ }^{3}$

We report the case of a 6-week-old infant who underwent brain computed tomography angiography (CTA) and magnetic resonance angiography (MRA). Neuroimaging indicated the presence of a basilar artery aneurysm, as well as bilateral subdural fluid collections and cerebral parenchymal injury. The presence of a cerebral aneurysm on imaging raised concern for abnormal vessels due to Menkes disease or other vascular disorder. Digital subtraction angiography (DSA), the gold standard technique for evaluation of cerebral aneurysm, was subsequently performed. DSA revealed no evidence of aneurysm and helped facilitate a diagnosis of AHT. This case highlights the potential for false positive CTA and MRA diagnoses of cerebral aneurysm and demonstrates how DSA played a role in the evaluation for AHT by enabling vascular abnormality to be ruled out.

\section{Case Presentation}

A 6-week-old male infant presented to a community hospital with a 2-day history of non-bloody, non-bilious vomiting following each feeding; irritability; and decreased oral intake. He was otherwise healthy, born full-term following an uncomplicated pregnancy and normal vaginal delivery with a normal newborn screen and no reported history of trauma.

Upon arrival, he was afebrile, with physical exam only remarkable for a bulging anterior fontanelle.
Ultrasound due to suspected pyloric stenosis was negative. A complete blood count (CBC) demonstrated leukocytosis with a white blood cell (WBC) count of 26 (5.0-19.0 K/ul), and a sepsis evaluation, including lumbar puncture, was initiated. Cultures, however, remained negative. He was admitted to the floor for treatment of dehydration and to rule out sepsis.

That day the patient continued to vomit, and the bulging anterior fontanelle persisted. On hospital day 2 a head CT demonstrated bilateral subdural fluid collections without midline shift or herniation (Figure 1a). A subsequent brain magnetic resonance imaging (MRI)/MRA redemonstrated these findings, as well as a diffusion abnormality in the right temporal lobe area and a suspicious $3 \times 4 \mathrm{~mm}$ basilar artery aneurysm (Figure 1b). At this time, the patient was sleepy but arousable, with movement of all extremities. He was transferred the same day to a children's hospital pediatric intensive care unit (PICU) for neurosurgical and child protection team evaluations.

On the evening of his arrival to the children's hospital, the patient had an observed episode of left eye twitching without convulsions. Electroencephalogram (EEG) monitoring revealed multiple subclinical seizures originating from the right occipital region. Skeletal survey was negative for fractures, and no bruising or other injury was noted upon physical exam. Ophthalmologic exam performed that evening revealed too numerous to count bilateral intra- and pre-retinal hemorrhages $(\mathrm{RH})$ extending to the periphery. CTA performed the following day redemonstrated previous findings, including the presence of a basilar aneurysm (Figure 1c). He was transferred to the floor 2 days after his admission and remained hospitalized for the next 2 weeks.

Regarding the patient's social history, he had been living with his mother and father, both of whom were in their twenties and were his primary caretakers. No other dependents lived in the home, although the patient's father had another child who lived with that child's mother. Both of 

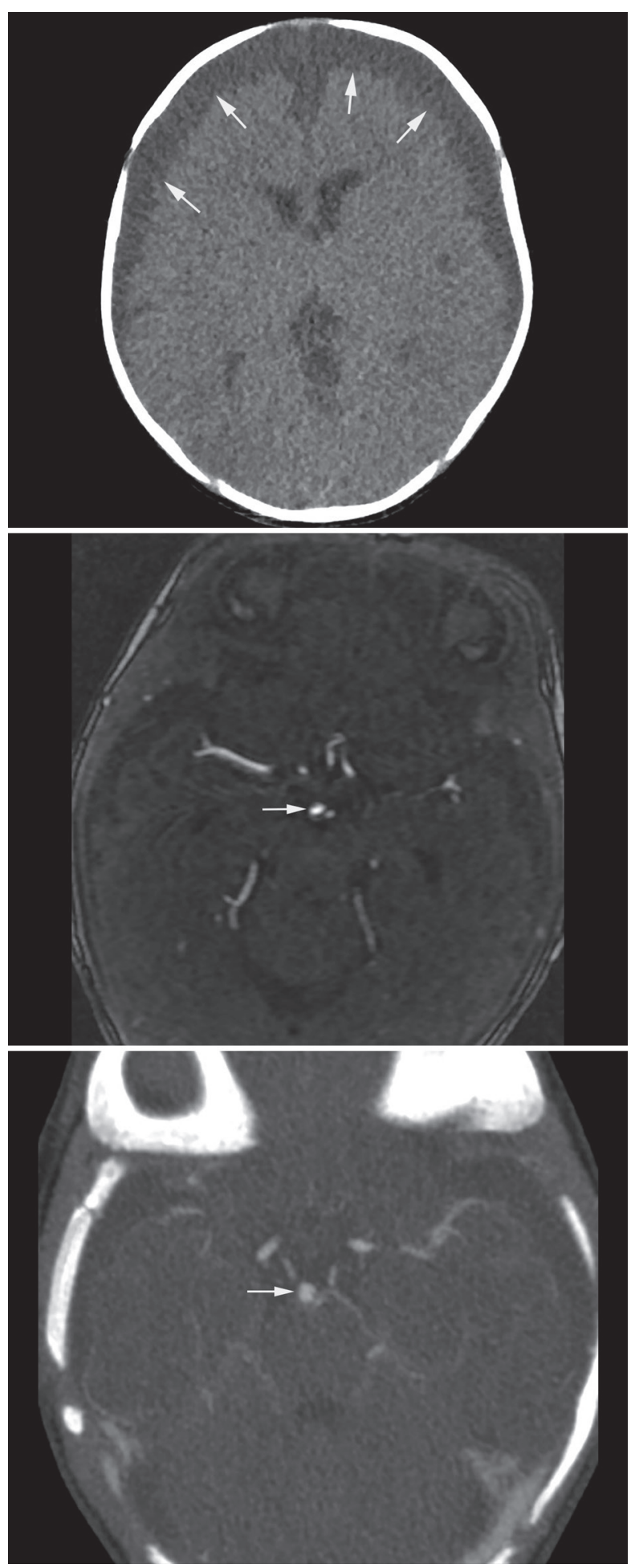

FIGURE 1. Head CT (1a) showing bilateral subdural collections (arrows) that are hypodense but slightly hyperdense to CSF. MRA (1 b) showing small area of flowrelated signal near the tip of the basilar artery (arrow) that is suspicious of an aneurysm. CTA (1c) showing focal bulbous appearance of the basilar artery (arrow), corresponding to the abnormality seen on prior MRI and MRA. his parents worked outside of the home with alternating shifts so that one parent was almost always with the patient. Occasionally, the mother's sister would care for the patient, but otherwise the patient had never been left alone with other caregivers. The patient had been exclusively formula fed since birth and was gaining weight appropriately. He was up to date on his vaccinations.

In regard to his family history, the patient's father reportedly had 2 strokes between the ages of 7-9. Hospital records for the father's medical care, however, were no longer extant. Due to this reported history, the uncertain significance of the basilar aneurysm, and the presence of diffusion abnormality and subdural collections, consults were placed with hematology to evaluate for coagulopathy or bleeding disorder and genetics to evaluate for the possibility of a metabolic disorder.

To visualize and further characterize the basilar aneurysm seen on MRA and CTA, neurosurgery recommended DSA, which was performed during the patient's hospital stay. DSA, however, revealed no evidence of a basilar or posterior cerebral artery aneurysm or arterial dissection (Figures 2a, 2b). Instead, proximal tortuosity of the posterior cerebral arteries, including the presence of a vertical loop, led to a bulbous appearance on prior neuroimaging, mimicking an aneurysm.

In addition to DSA, a workup for possible hematologic and metabolic causes of the patient's imaging findings was negative. The patient's bilateral subdural collections and right temporal diffusion abnormality with no hematologic or metabolic cause and no trauma history, as well as his multilayered, too numerous to count $\mathrm{RH}$ out to the periphery, were considered to be a result of AHT.

\section{Discussion}

Pediatric abusive head trauma is defined by the US Centers for Disease Control and Prevention as "injury to the skull or intracranial contents of an infant or young child ( $<5$ years of age) due to inflicted blunt impact and/or violent shaking." 

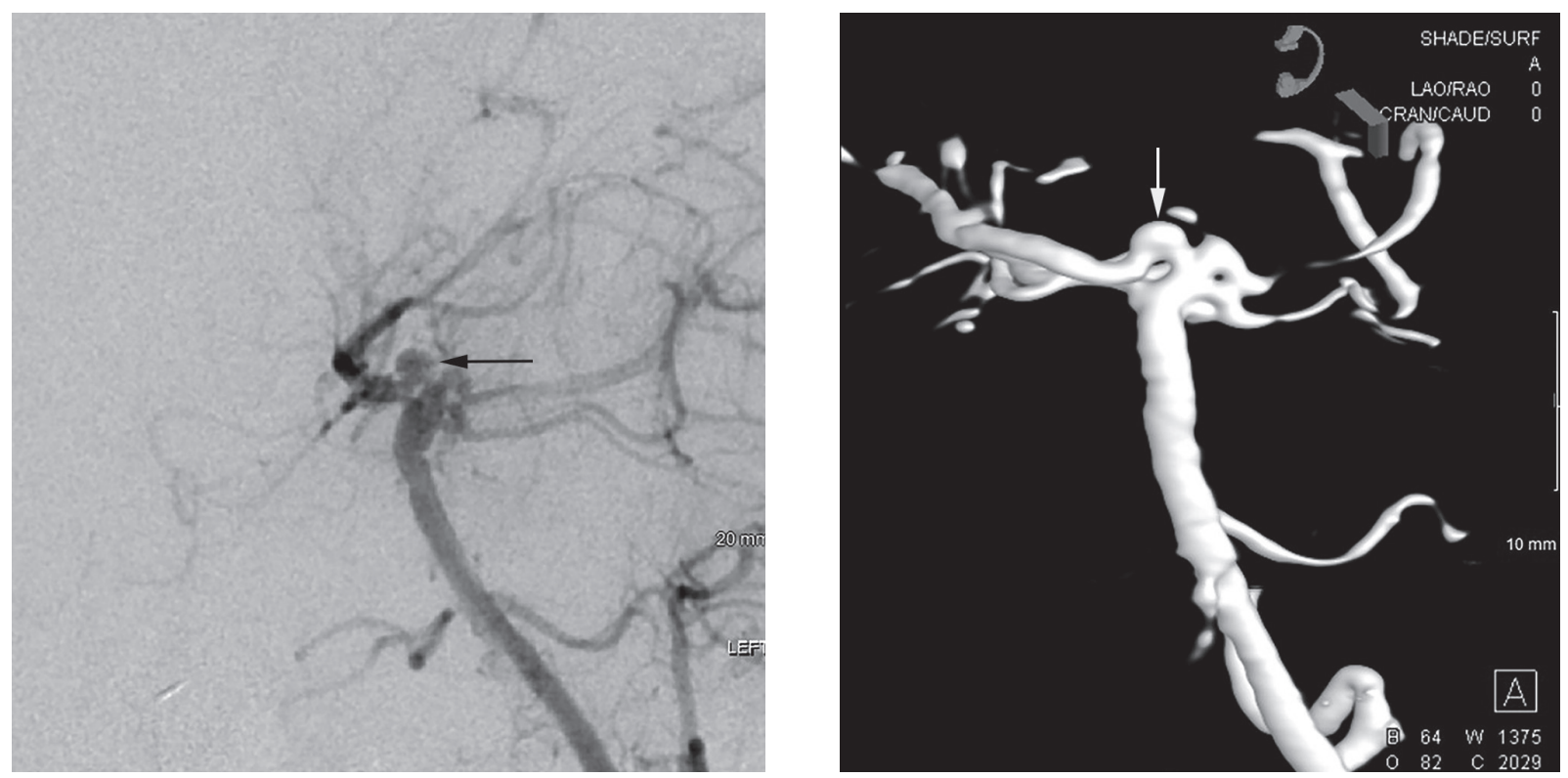

FIGURE 2. DSA-left vertebral artery injection (2a) and 3D rendered image (2b) showing focal bulbous appearance of the proximal right posterior cerebral artery (arrows). This was thought to be responsible for the MRA and CTA findings. No aneurysm was found.

Symptoms are typically nonspecific and include vomiting, irritability, lethargy, and seizures..$^{2,5}$ Among victims of AHT, subdural hemorrhage (SDH) is the most frequently observed intracranial imaging finding. ${ }^{6,7}$ Stroke is also commonly observed in pediatric AHT, with one study finding that $28 \%$ of children diagnosed with AHT over a 7-year period at a US children's hospital had evidence of at least one cerebrovascular accident (CVA). ${ }^{8}$ In addition, too numerous to count $\mathrm{RH}$ that extend to the periphery in the absence of an underlying medical condition such as glutaric aciduria, coagulopathy, or oncologic processes and no history of trauma are highly specific for AHT., 9,10

In cases of suspected AHT, potential nonabusive causes of the findings are considered, including birth-related trauma; vascular abnormalities; and hematologic, metabolic, connective tissue, and other genetic disorders. Workup for suspected AHT is based on patient presentation, with the American Academy of Pediatrics (AAP) recommending consultation with a subspecialist in child abuse pediatrics as well as subspecialists in neurology, neurosurgery, radiology, and ophthalmology, among other fields, based on the clinical presentation. ${ }^{11}$ In this case, the presence of what appeared to be a basilar artery aneurysm on CTA and MRA raised concern for an underlying genetic or vascular cause of the patient's intracranial and retinal findings.

Birth-related trauma was considered as a potential cause of the subdural collections. However, birth-related SDH typically resolves without becoming chronic, with one study reporting that 93.8\% of SDH observed within 72 hours of childbirth had resolved within 1 month and all had resolved by 3 months. ${ }^{12}$ In this case, imaging performed at 16 weeks showed the persistence of bilateral fluid collections.

Other diagnoses that were considered include Menkes disease, which can cause tortuosity of cerebral vessels and intracerebral hemorrhage. ${ }^{13}$ Ceruloplasmin and serum copper levels, as well as microscopic hair analysis, however, were within normal limits. Additionally, glutaric aciduria type 1 was considered, as it can present with SDH, RH, seizure, and encephalopathy. ${ }^{14,15}$ Urine organic acids, however, were within normal limits. 
In this case, DSA distinguished arterial tortuosity from aneurysm and enabled a vascular abnormality to be ruled out as a possible contributing factor to this patient's presentation. DSA is an invasive technique in which a catheter is introduced into the vasculature being studied and imaging is taken before and after the delivery of contrast. Precontrast imaging is then subtracted digitally from postcontrast images, which allows vessels that are filled with contrast to be viewed independently. ${ }^{16,17}$ DSA provides high magnification images of up to 0.1-0.2 $\mathrm{mm}$ and can be further enhanced by 3-dimensional images created via rotational angiography..$^{18}$ DSA is the gold standard diagnostic approach for cerebral aneurysm, yet its invasiveness and complication rate (0.1\% to $2.5 \%)$ remain significant drawbacks. ${ }^{18-21}$

The 2 main alternative approaches are CTA and MRA. CTA has become a common screening tool for intracranial aneurysm as it offers a fast, minimally invasive option with relatively high specificity and sensitivity. ${ }^{22}$ Principle drawbacks of CTA include difficulty visualizing aneurysms smaller than $3 \mathrm{~mm}$, difficulty visualizing aneurysms in the context of cerebrovascular disease, difficulty distinguishing overlapping vessels from an aneurysm, and mistaking venous structures and infundibuli for an aneurysm. 18,19,22 Studies have reported a range of sensitivities and specificities for the diagnosis of intracranial aneurysm using CTA, potentially a result of the small sample sizes used in many studies. ${ }^{22}$ A recent retrospective study that included a larger cohort consisting of 1,366 patients reported per-aneurysm sensitivities of $95.2 \%$ and $95.4 \%$ and per-aneurysm specificities of $96.6 \%$ and $97.0 \%$, results similar to those reported in a prior meta-analysis of CTA used to diagnose intracranial aneurysm.22,23 In comparison to CTA, MRA has the advantages of no exposure to radiation or iodinated contrast. In one meta-analysis, MRA had a sensitivity of $95 \%$ and specificity of $89 \%$ for the diagnosis of intracranial aneurysm, with a false positive rate of about $5.6 \% .{ }^{24}$
DSA is not a standard component of an evaluation for AHT. However, given the false positive rates of CTA and MRA, when the finding of an aneurysm using these techniques seems not to fit with the patient's constellation of other findings, consideration should be given to the use of DSA to ascertain whether or not an aneurysm is truly present.

Limitations of this study include being based on a single clinical case as well as the reliability of the history provided by the patient's caregivers.

\section{Conclusion}

Given the emotional, social, and legal ramifications of an AHT diagnosis, consideration of other, nonabusive etiologies is essential. This case highlights the use of DSA as a tool in the evaluation of suspected abusive head trauma. Although the aneurysm observed on CTA and MRA could not have accounted for the patient's subdural collections, diffusion abnormality, or RHs, it raised suspicion for an underlying vascular abnormality that could have contributed to the patient's findings. Vascular abnormality broadened the differential diagnosis for the patient's subdural collections and RHS and was excluded only after negative results were obtained by DSA.

\section{References}

1. Niederkrotenthaler $\mathrm{T}, \mathrm{Xu} \mathrm{L}$, Parks $\mathrm{SE}$, Sugerman DE. Descriptive factors of abusive head trauma in young children-United States, 2000-2009. Child Abuse Neg/. 2013;37(7):446-455. doi:10.1016/ j.chiabu.2013.02.002

2. Greeley CS. Abusive head trauma: a review of the evidence base. Am J Roentgenol. 2015;204(5):967973. doi:10.2214/AJR.14.14191

3. Parks SE, Kegler SR, Annest JL, Mercy JA. Characteristics of fatal abusive head trauma among children in the USA, 2003-2007: an application of the CDC operational case definition to national vital statistics data. Inj Prev. 2012;18(3):193-199. doi:10.1136/ injuryprev-2011-040128

4. Parks SE, Annest JL, Hill HA, Karch DL. Pediatric Abusive Head Trauma: Recommended Definitions for Public Health Surveillance and Research. Atlanta, GA: Centers for Disease Control and Prevention. 
https://www.cdc.gov/violenceprevention/pdf/ pedheadtrauma-a.pdf. Published 2012. Accessed December 26, 2016.

5. Jenny C, Hymel KP, Ritzen A, Reinert SE, Hay TC. Analysis of missed cases of abusive head trauma. JAMA. 1999;281(7):621-626. doi:10.1001/jama.281 .7 .621

6. Hedlund G. Abusive head trauma: extra-axial hemorrhage and nonhemic collections. In: Kleinman P, ed. Diagnostic Imaging of Child Abuse. 3rd ed. Cambridge, UK: Cambridge University Press; 2015:394-452.

7. Pollanen MS, Smith CR, Chiasson DA, Cairns JT, Young J. Fatal child abuse-maltreatment syndrome: a retrospective study in Ontario, Canada, 1990-1995. Forensic Sci Int. 2002;126(2):101-104. doi:10.1016/S0379-0738(02)00008-7

8. Khan NR, Fraser BD, Nguyen V, et al. Pediatric abusive head trauma and stroke. J Neurosurg Pediatr. 2017;20:183-190. doi:10.3171/2017.4.PEDS16650

9. Binenbaum G, Chen W, Huang J, Ying GS, Forbes BJ. The natural history of retinal hemorrhage in pediatric head trauma. J AAPOS. 2016;20(2):131-135. doi:10.1016/j.jaapos.2015.12.008

10. Binenbaum G, Forbes BJ. The eye in child abuse: key points on retinal hemorrhages and abusive head trauma. Pediatr Radiol. 2014;44(4):571-577. doi:10.1007/s00247-014-3107-9

11. Christian CW, Block R, Committee on Child Abuse and Neglect. Abusive head trauma in infants and children. Pediatrics. 2009;123(5):1409-1411. doi:10.1542/peds.2009-0408

12. Rooks VJ, Eaton JP, Ruess L, Petermann GW, KeckWherley J, Pedersen RC. Prevalence and evolution of intracranial hemorrhage in asymptomatic term infants. AJNR Am J Neuroradiol. 2008;29(6):10821089. doi:10.3174/ajnr.A1004

13. Ropper AH, Samuels MA, Klein JP. Inherited metabolic diseases of the nervous system. In: Adams and Victor's Principles of Neurology. 10th ed. New York, NY: McGraw-Hill; 2014. http://accessmedicine . mhmedical.com/content.aspx?aid=57625274. Accessed April 25, 2019.

14. Hartley LM, Khwaja OS, Verity CM. Glutaric aciduria type 1 and nonaccidental head injury. Pediatrics.
2001;107(1):174-175. doi:10.1542/peds.107.1 .174

15. Morris AA, Hoffmann GF, Naughten ER, Monavari AA, Collins JE, Leonard JV. Glutaric aciduria and suspected child abuse. Arch Dis Child. 1999;80(5):404-405.

16. Okamoto K, Ito J, Sakai K, Yoshimura S. The principle of digital subtraction angiography and radiological protection. Interv Neuroradiol. 2000;6(Suppl 1):2531. doi:10.1177/15910199000060S102

17. Harrington DP, Boxt LM, Murray PD. Digital subtraction angiography: overview of technical principles. AJR Am J Roentgenol. 1982;139(4):781-786. doi:10.2214/ajr.135.6.1316

18. Pradilla G, Wicks RT, Hadelsberg $U$, et al. Accuracy of computed tomography angiography in the diagnosis of intracranial aneurysms. World Neurosurg. 2013;80(6):845-852. doi:10.1016/j.wneu.2012.12 .001

19. Kim JH, Cheong JH, Bak KH, Kim CH, Kim JM. Venous loop mimicking middle cerebral artery bifurcation aneurysm on computed tomographic angiographycase report. Surg Neurol. 2006;66(5):524-526. doi:10.1016/j.surneu.2006.02.035

20. Robertson RL, Chavali RV, Robson CD, et al. Neurologic complications of cerebral angiography in childhood moyamoya syndrome. Pediatr Radiol. 1998;28(11):824-829. doi:10.1007/s002470050474

21. Pettersson H, Fitz CR, Harwood-Nash DC, Chuang S, Armstrong E. latrogenic embolization: complication of pediatric cerebral angiography. AJNR Am J Neuroradiol. 1981;2(4):357-361.

22. Yang ZL, Ni QQ, Schoepf UJ, et al. Small intracranial aneurysms: diagnostic accuracy of CT angiography. Radiology. 2017;285(3):162290. doi:10.1148/radiol .2017162290

23. Menke J, Larsen J, Kallenberg K. Diagnosing cerebral aneurysms by computed tomographic angiography: meta-analysis. Ann Neurol. 2011;69(4):646654. doi:10.1002/ana.22270

24. Sailer AM, Wagemans BA, Nelemans PJ, de Graaf R, van Zwam WH. Diagnosing intracranial aneurysms with MR angiography: systematic review and metaanalysis. Stroke. 2014;45(1):119-126. doi:10.1161/ STROKEAHA.113.003133 